\title{
On dendrogram-based measures of functional diversity
}

\author{
János Podani and Dénes Schmera
}

Podani, J. and Schmera, D. 2006. On dendrogram-based measures of functional diversity. - Oikos 115: 179-185.

\begin{abstract}
Euclidean distance is commonly involved in calculating functional diversity (FD), for example, in measures based on dendrogram branch lengths. We point out that this function is inappropriate in many cases and that the choice of clustering method is more crucial than earlier thought. Gower's formula and UPGMA clustering are suggested here as a standard combination of techniques for calculating FD. The advantage of Gower's measure is its suitability to a mixture of scale types and its tolerance to missing values. Examples demonstrate that UPGMA clustering is more robust and has a better goodness of fit to dissimilarities than complete and single linkage classifications. In addition, we propose that the effect of individual species on FD is best evaluated by species removals and subsequent comparisons of tree length values. The influence of each functional trait is optimally judged by considering both dendrogram length and topological changes.
\end{abstract}

J. Podani (podani@ludens.elte.hu),Dept of Plant Taxonomy and Ecology,Eötvös Loránd Univ., Pázmány P. s. 1/c, Budapest, HU-1117, Hungary. - D. Schmera, Dept of Zoology, Plant Protection Inst., Hungarian Academy of Sciences, POB 102, Budapest, HU-1525, Hungary.

The measurement of functional diversity (FD) has been in the focus of ecological research for several years (Tilman 2001, Mason et al. 2003, 2005, Naeem and Wright 2003, Petchey et al. 2004, Botta-Dukát 2005, Mouillot et al. 2005, Ricotta 2005). One of the recent approaches applies cluster analysis of species based on Euclidean distances calculated from a matrix of functional traits, and then uses the sum of branch lengths of the dendrogram (here called the tree length) as a multivariate measure of functional diversity (Petchey and Gaston 2002). This is done with the implicit assumption that tree length is an appropriate measure of diversity (Faith 1992) and is therefore preferable against the sum of distances suggested by Walker et al. (1999). Trait matrices are not always homogeneous in measurement scale, because a mixture of possible data types (nominal, ordinal, interval and ratio, Anderberg 1973) may appear simultaneously. Also, trait matrices are often incomplete, e.g. when no information is available on a given trait for a given species or when it is illogical to define a character for a subset of species (e.g. leaf size for lichens). Euclidean distance does not apply to the nominal and ordinal scale, however, and previous standardization by standard deviation is also completely meaningless for these two scale types. Missing scores cannot be handled by Euclidean distance either, and replacement of missing scores by zero obviously does not solve the problem, because zero is a misleading score in this case. Furthermore, Euclidean distance is influenced by the number of functional characters used, which is also considered as a disadvantage (Mason et al. 2005). Another difficulty with the cluster-analysis based approach is that the shape of the dendrogram and the branch lengths may differ considerably with the clustering strategy applied. In other words, the robustness of hierarchical classification to the clustering method, e.g. group average (UPGMA), single linkage

Accepted 24 April 2006

Subject Editor: Owen Petchey

Copyright (C) OIKOS 2006

ISSN 0030-1299

OIKOS 115:1 (2006) 
(SL) and complete linkage (CL), is strongly casedependent (Podani 2000).

The suggestion by Petchey and Gaston (2002) that for the single-species case FD is to be positive, with 0 being "reserved for communities without any species" also requires attention. In addition to the fact that no diversity can be defined for something that does not exist, we note that all diversity formulations return zero diversity for a community in which all individuals represent the same species (e.g. Shannon entropy), and we feel that FD cannot be exception. Petchey and Gaston's definition derives from the proposition that FD for a subset of species should be based on branch removals in a dendrogram of the full set of species. Clearly, some alternative solution is needed for evaluating species contributions to FD in order to avoid conflict with traditional diversity concepts.

First we discuss the methodological problems associated with trait distances and put forward some suggestions as to the standardized use of data analysis methodology. We propose the use of the dissimilarity version of Gower's formula (Gower 1971, modified by Podani 1999) which allows the presence of a mixture of all variable types and tolerates missing values as well. We demonstrate case dependence of three cluster analysis methods and conclude that UPGMA be used as the standard method for clustering from trait matrices and in turn calculating FD in terms of tree length. We propose, further, that the effect of a species or a species group upon FD can be expressed by obtaining a dendrogram with and without this species or group, respectively, and comparing the tree lengths of these two dendrograms. We also address the question of how to measure the contribution of a single trait to FD. We suggest that this is obtained readily by calculating the dissimilarity matrix among species with and without a selected trait, followed by the derivation of trees for both cases. Comparison of the two dendrograms in terms of tree length allows measuring how FD is affected by the selected trait. Actual data sets taken from the literature are used throughout as illustrative examples.

\section{Methodological issues}

\section{The data type problem}

The five case studies appearing in Petchey and Gaston's (2002) meta-analysis sufficiently demonstrate the heterogeneity of data ecologists may be faced with when calculating FD. Three data sets (Holmes et al. 1979, Jaksić and Medel 1990 and Muñoz and Ojeda 1997) are homogeneous as to the measurement scale of the traits: all variables are percentages. To such variables, standardization by variance and subsequent use of Euclidean distance, as suggested by Holmes et al. (1979) and Petchey and Gaston (2002) are in fact straightforward. The other two data sets, however, pose several problems. In the Patagonian forbs data presented by Golluscio and Sala (1993), there are three variables measured on the ratio scale (Anderberg 1973), but one of them ("maximum sprouting depth") has several missing values. There is another variable ("mesophytic degree") with three states: mesophytic (coded by 1 ), intermediate (0) and xerophytic $(-1)$. This is apparently an ordinal variable (only the ordering of possible values is meaningful; Anderberg 1973, Podani 2005), because there is no information on how much the different states differ from one another. Two variables, expressed in months, are circular such that December is coded by 12 and January by 1 . However, if two species are close in some phenological phase in these two months within the same vegetation period, then their difference is in fact 1 , rather than 11 which would result from the inadvertent application of Euclidean distance. The authors do not provide information on how these missing values, ordinal and circular variables were handled. In Chapin et al. (1996), the arctic vegetation data are even more heterogeneous in measurement scale: 10 ratioscale, 6 ordinal, and 5 nominal variables are included, three ratio scale variables with quite a few missing scores. Leaf width represents a trait which is illogical for certain taxa, such as lichens, and is therefore potential source of missing scores. An example for the nominal scale from this study is seed dormancy with the following states: "0-germinate immediately, 1-germinate after one winter, 2-germinate with difficulty". These variable states could be coded arbitrarily by any other numbers in any sequence, so that Euclidean distance is ill-defined for such variables. Again, there is no information in the respective papers on how these were handled in calculating Euclidean distance and subsequent UPGMA clustering.

\section{Calculating dissimilarities from mixed data}

There has long been a formula which solves most of the problems mentioned above: Gower's (1971) similarity function to mixed data types can handle ratio, interval and nominal scale variables simultaneously such that missing values are also appropriately considered. Its extension (Podani 1999) allows inclusion of ordinal variables as well in the data. This modified function implies standardization of each ordinal, interval and ratio scale variable by range, whereas the range for nominal variables is unity by definition, so that all variables are equally weighted when calculating similarity. This is certainly more elegant than standardizing nominal and ordinal variables to unit variance, which is a mathematically inappropriate operation for these 
scale types. Of course, the formula may be applied to data containing only ratio scale variables, by noting that standardization of each variable by its range often gives similar results to standardization by variance. Gower's formula has a range of $[0,1]$ irrespective of the number of variables, so that tree length is unaffected by the number of traits, which is considered an advantage by Mason et al. (2005). Thus, we suggest the use of the following dissimilarity version of Gower's formula

$\mathrm{d}_{\mathrm{jk}}=\frac{\sum_{\mathrm{i}=1}^{\mathrm{n}} \mathrm{w}_{\mathrm{ijk}} \mathrm{s}_{\mathrm{ijk}}}{\sum_{\mathrm{i}=1}^{\mathrm{n}} \mathrm{w}_{\mathrm{ijk}}}$

where $\mathrm{n}$ is the number of functional traits (variables). The weight $\mathrm{w}_{\mathrm{ijk}}=0$ if the comparison of species $\mathrm{j}$ and $\mathrm{k}$ is not allowed for variable $\mathrm{i}$ owing to missing data, otherwise $w_{i j k}=1$. Furthermore, $s_{i j k}$ is a measure of disagreement between species $\mathrm{j}$ and $\mathrm{k}$ for variable $\mathrm{i}$. Its definition depends on the type of scale as described below.

For nominal variables:

$\mathrm{s}_{\mathrm{ijk}}=1$, if $\mathrm{x}_{\mathrm{ij}} \neq \mathrm{x}_{\mathrm{ik}}$

$\mathrm{s}_{\mathrm{ijk}}=0$, if $\mathrm{x}_{\mathrm{ij}}=\mathrm{x}_{\mathrm{ik}}$

where $x_{i j}$ is the raw data score for variable $\mathrm{i}$ and species $\mathrm{j}$.

For ordinal variables, all scores, $\mathrm{x}_{\mathrm{ij}}$, are replaced by their ranks, $r_{i j}$. For example, if $x_{i j}=6$ is the fifth value in the ascending rank order of all scores for variable $i$, then $r_{i j}=5$. Disagreement between two species for variable $i$ is obtained by

$\mathrm{s}_{\mathrm{ijk}}=\left|\mathrm{r}_{\mathrm{ij}}-\mathrm{r}_{\mathrm{ik}}\right| /\left[\max \left\{\mathrm{r}_{\mathrm{i}}\right\}-\min \left\{\mathrm{r}_{\mathrm{i}}\right\}\right]$.

Alternatively, $s_{i j k}$ is defined in terms of the number of elementary steps (interchanges) neded to put an object with the same value as $\mathrm{x}_{\mathrm{ij}}$ into a position of another object which has the same value as $\mathrm{x}_{\mathrm{ik}}$ in the rank order (Podani 1999, for more details).

For variables measured on the interval and ratio scale, we have

$\mathrm{s}_{\mathrm{ijk}}=\left|\mathrm{x}_{\mathrm{ij}}-\mathrm{x}_{\mathrm{ik}}\right| /\left[\max \left\{\mathrm{x}_{\mathrm{i}}\right\}-\min \left\{\mathrm{x}_{\mathrm{i}}\right\}\right]$

The denominator in Eq. 4 and 5 is the range for variable $\mathrm{i}$, therefore standardization to unit range is implied. To make a choice between the two formulations for ordinal variables, we suggest to examine whether ties (equal values) occur in the ranks. If they do not appear, Eq. 4 is suggested. Otherwise, ties can be accounted for and their distorting effect removed by the interchange version of the coefficient.

The value of formula (1) is zero when the two species being compared are identical in all traits, whereas the value of 1 is obtained when the two species maximally differ in all traits. Note that tolerance to missing data does not mean that excessive amounts of missing scores are allowed. For example, the denominator in Eq. 1 becomes zero when none of the variables is observed for both species, a clearly undesirable situation.

\section{Clustering from distance matrices}

Cluster analysis from distance (dissimilarity) matrices has long been the most widely used procedure of representing distance structures in terms of trees. Wellknown strategies of hierarchical clustering, such as group average (UPGMA), single linkage (SL) and complete linkage (CL, for more procedures, see Gordon 1999, Podani 1989) produce a classification in form of a dendrogram, with meaningful weights on the branches (branch lengths). Petchey and Gaston (2002) define FD as the tree length (total branch length) of the dendrogram, commenting that "qualitative relations between FD, species richness, and community composition" are robust to the clustering strategy used. However, this is usually not the case, because CL and SL represent endpoints of a "methodological gradient" between space-dilating and space-contracting methods, with UPGMA somewhere in between (Gordon 1999). Space-dilating methods are known to indicate clusters even in cases without group structure in the data, whereas space contracting strategies may leave some clusters undetected even if they are apparent. UPGMA represents a good compromise between the two extremes, and thus it is suggested to be the only clustering method for use in calculating FD. We shall demonstrate its superiority over the other two procedures by using the cophenetic correlation function (Sneath and Sokal 1973, Podani 2000) which measures how the original dissimilarity structure is preserved by the dendrogram. Blackburn et al. (2005) also used this method for finding the best clustering strategy for calculating FD in mammal assemblages. Cophenetic correlation is a formal application of the product moment correlation coefficient for comparing two matrices value by value. The first one is the dissimilarity matrix, $D=\left\{\mathrm{d}_{\mathrm{jk}}\right\}$ whereas each value $h_{j k}$ in the second matrix $H$ represents the lowest hierarchical level where the given pair of species belong to the same cluster.

\section{Species contributions}

The caption to Fig. 1 in Petchey and Gaston (2002) suggests that after removing a group of species or a single species from the dendrogram, the length of the remaining dendrogram is obtained by distracting those lengths that pertain to the removed species. If a single species remains, those authors propose to use the sum of branch lengths from this object to the root of the original dendrogram so that the FD of a single species community is a positive value, with 0 being "reserved for communities without species". This suggestion cannot 
be accepted by us for three reasons. First, the total branch length between the object and the root of the dendrogram is the same value for all objects (i.e. the highest hierarchical level in the dendrogram), so this "single species" diversity value does not make much sense. Second, for one species the dendrogram in fact collapses into a single vertex, and the branch length will be zero, so FD must also be zero if we want to be consistent with its definition. Third, in almost all other definitions of ecological diversity the basic requirement is that diversity is 0 for the single species case (e.g. for Shannon index and Gini-Simpson index, the trivial exception being 1 for species richness). If a community is composed of a single species, then it is entirely homogeneous functionally, and reserving $\mathrm{FD}=0$ for a community "without species" is unwarranted. As a different approach to the problem of measuring how FD is influenced by the removal of species $j$, we suggest here performing cluster analysis and calculating FD without species $\mathrm{j}$ and then comparing the result with the FD of the full set of species. Doing so separately for each species allows the identification of species that play crucial role in affecting functional diversity of the community. Two or more species may also be removed at a time, but this possibility will not be examined in this paper.

\section{Contributions of single traits}

Individual traits or variables are equally weighted a priori by the Gower index, and therefore they receive the same weight in calculating total branch lengths, i.e. FD as well. It does not mean, of course, that a posteriori all traits will be equal. To see how FD is influenced by each trait we suggest a procedure similar to the one described above. In this, cluster analysis is performed on reduced data sets, with one variable omitted at a time. Then, the resulting tree length values are compared to the tree length based on the full set of variables. The difference between the values will give us a measure of trait contribution to tree length. Again, a similar procedure can be applied to the removal of groups of traits, a possibility not examined here.

\section{Examples}

\section{Calculating dissimilarities and FD}

The five data sets evaluated by Petchey and Gaston (2002) were reanalyzed using the Gower formula as the dissimilarity function between species. Three data sets (Holmes et al. 1979, Jaksić and Medel 1990, Muñoz and Ojeda 1997) required no specific treatment, whereas in the Patagonian forbs data set (Golluscio and Sala 1993) one ordinal and five ratio scale variables were distinguished, and missing values ignored from the comparisons, thanks to the flexibility of Gower formula. The same applies to missing scores in the arctic vegetation data set (Chapin et al. 1996), in which five nominal, six ordinal variables were distinguished from the remaining ratio scale variables. Circularity of phenological variables in the Patagonian forbs data was also corrected for. The resulting dissimilarity matrices were evaluated by group average, single linkage, and complete linkage clustering. Cluster analyses were performed by the SYN-TAX 2000 program package (Podani 2001), whereas a small routine was written for calculating tree lengths and cophenetic correlations based on distances and dendrogram data saved by SYN-TAX. The results are not reproduced here, because the dendrograms are methodologically incomparable to the published alternatives, especially for the arctic vegetation and Patagonian forbs.

\section{Fitting to dissimilarities and dendrogram length}

The goodness of fit of each resulting dendrogram to the dissimilarities as measured by the cophenetic correlation is summarized in Table 1. The UPGMA method outperforms the other procedures in all cases, as expected on theoretical grounds. It is not surprising either that CL produces a better fit to the distances than SL in four cases, but the inconsistency for these two procedures is demonstrated well by the higher cophenetic correlation obtained for SL for the intertidal fish data. The difference between the performance of these two clustering methods is almost negligible for the Patagonian forbs data.

Trends in tree lengths are more or less consistent over the five datasets (Table 2). The shortest values are achieved by SL, then UPGMA and finally CL.

Table 1. Cophenetic correlations between the dissimilarity matrix and hierarchical levels for five data sets and three clustering methods.

\begin{tabular}{lccrr}
\hline Data & No. of species & CL & UPGMA & SL \\
\hline Arctic vegetation (Chapin et al. 1996) & 37 & 0.740 & 0.771 & 0.722 \\
Patagonian forbs (Golluscio and Sala 1993) & 24 & 0.664 & 0.708 & 0.662 \\
Insectivorous birds (Holmes et al. 1979) & 22 & 0.770 & 0.795 & 0.598 \\
Intertidal fish (Muñoz and Ojeda 1997) & 13 & 0.702 & 0.890 & 0.865 \\
Predatory vertebrates (Jaksić and Medel 1990) & 11 & 0.759 & 0.773 & 0.722 \\
\hline
\end{tabular}


Table 2. Tree lengths for five data sets and three clustering methods.

\begin{tabular}{lccrr}
\hline Data & No. of species & CL & UPGMA & SL \\
\hline Arctic vegetation (Chapin et al. 1996) & 37 & 6.55 & 5.17 & 3.77 \\
Patagonian forbs (Golluscio and Sala 1993) & 24 & 5.30 & 3.91 & 2.58 \\
Insectivorous birds (Holmes et al. 1979) & 22 & 4.55 & 4.00 & 3.31 \\
Intertidal fish (Muñoz and Ojeda 1997) & 13 & 3.25 & 2.77 & 2.17 \\
Predatory vertebrates (Jaksić and Medel 1990) & 11 & 3.31 & 2.80 & 2.38 \\
\hline
\end{tabular}

Comparison of values along with methods is less interesting, however, because tree lengths reflect no more than the mathematical properties of the three clustering algorithms. i.e., a transition from spacecontracting to space-dilating methods. Comparisons over data sets indicate, on the other hand, that tree length, and therefore FD, and the number of objects are largely positively correlated. The observation that the SL and UPGMA dendrograms are longer for insectivorous birds (22 species) than for Patagonian forbs ( 24 species) nevertheless indicates that this overall positive correlation may be overridden by differences in functional diversity: lower tree length for more species strongly supports the view that the Patagonian forbs dataset is functionally less diverse than the insectivorous bird data (except for CL). Comparison of tree lengths for the predatory vertebrates (11 species) and intertidal fish data (13 species) provides similar results, i.e. fewer species produce higher diversity and in this case the three clustering procedures are consistent with one another.

\section{Species contributions}

The effect of species removal upon tree length was examined based on the intertidal fish data, which include 13 fish species described in terms of 16 prey groups as functional traits. The direction of change was almost always the same for the three clustering methods: for four species, tree length increased after data reduction, whereas the removal of each of seven other species

Table 3. The effect of single species removals on tree length for the intertidal fish data and three clustering methods. Increased values are emphasized by boldface. Minima are coded by *, maxima by ^ for each method.

\begin{tabular}{lccc}
\hline Species removed & CL & UPGMA & SL \\
\hline none & 3.245 & 2.766 & 2.171 \\
1 & 3.211 & 2.703 & 2.165 \\
2 & $\mathbf{3 . 3 1 4}$ & $\mathbf{2 . 8 2 4}$ & $\mathbf{2 . 2 1 8}$ \\
3 & $\mathbf{3 . 3 5 9}$ & $\mathbf{2 . 7 9 3}$ & $\mathbf{2 . 2 8 2}$ \\
4 & $2.967^{*}$ & $2.574^{*}$ & $\mathbf{2 . 1 8 1}$ \\
5 & $\mathbf{3 . 3 3 6}$ & $\mathbf{2 . 9 3 3}$ & $\mathbf{2 . 3 4 2}$ \\
6 & 3.199 & 2.726 & $\mathbf{2 . 1 8 0}$ \\
7 & 3.181 & 2.721 & 2.119 \\
8 & 3.109 & 2.692 & 2.136 \\
9 & 3.122 & 2.688 & $2.094^{*}$ \\
10 & 3.164 & 2.744 & 2.126 \\
11 & 3.165 & 2.725 & 2.111 \\
12 & $\mathbf{3 . 3 1 5}$ & $\mathbf{2 . 8 0 5}$ & $\mathbf{2 . 1 9 2}$ \\
13 & 3.165 & 2.719 & 2.121 \\
\hline
\end{tabular}

resulted in a decrease of FD (Table 3). The exceptions are species 4 and 6 whose removal decreased dendrogram length only for UPGMA and CL. In case of UPGMA, the maximum increase was detected for species 5 (Auchenionchus microcirrhis), followed by species 2 (Hypsoblennius sordidus), whereas the maximum decrease was observed for species 4 (Tripterygion cunninghami). These changes never reached $10 \%$ of tree length, however. Examination of the raw data shows that species whose removal leads to increased FD have a much more balanced diet than those for which diversity decrease was observed. For example, Auchenionchus microcirrhis has three large values (decapod crabs $40.7 \%$, teleosts $-30.9 \%$, crustaceans $-16.4 \%$ ) just like Hypsoblennius sordidus (cirripedian cirri - $34.7 \%$, unidentified animal prey $-31.7 \%$ and macroalgae $-18.4 \%$ ) whereas Tripterygion cunninghami mostly feeds on a single prey group, the amphipods $(78.2 \%)$ with all other prey much below $10 \%$ each.

\section{Contributions of single traits}

Removal of one trait at a time had less substantial influence on FD than species omissions (Table 4). Both positive and negative changes occurred which were always smaller than $5 \%$ of tree length. The three methods were less consistent in this case than for species removals, and tree length increased for CL many more

Table 4. The effect of single trait removals on tree length for the intertidal fish data and three clustering methods. Increased values are emphasized by boldface. Minima are coded by *, maxima by ^ for each method.

\begin{tabular}{lccc}
\hline Trait removed & CL & UPGMA & SL \\
\hline none & 3.245 & 2.766 & 2.171 \\
1 & $\mathbf{3 . 3 2 4}$ & 2.755 & 2.116 \\
2 & 3.214 & 2.686 & 2.083 \\
3 & $\mathbf{3 . 3 4 3}$ & $\mathbf{2 . 7 9 4}$ & 2.137 \\
4 & $\mathbf{3 . 2 7 3}$ & $\mathbf{2 . 7 6 9}$ & $\mathbf{2 . 1 9 5}$ \\
5 & $\mathbf{3 . 2 7 0}$ & 2.688 & 2.040 \\
6 & 3.214 & 2.702 & $2.029 *$ \\
7 & $3.109^{*}$ & $2.635^{*}$ & 2.162 \\
8 & $\mathbf{3 . 3 7 6}$ & $\mathbf{2 . 8 1 9}$ & $\mathbf{2 . 2 2 6}$ \\
9 & 3.178 & 2.733 & 2.168 \\
10 & $\mathbf{3 . 3 1 8}$ & 2.741 & 2.108 \\
11 & $\mathbf{3 . 2 7 2}$ & 2.748 & 2.152 \\
12 & 3.217 & 2.732 & 2.171 \\
13 & $\mathbf{3 . 2 5 0}$ & $\mathbf{2 . 7 8 9}$ & $\mathbf{2 . 2 1 3}$ \\
14 & $\mathbf{3 . 3 1 9}$ & $\mathbf{2 . 8 0 8}$ & $\mathbf{2 . 2 1 0}$ \\
15 & $\mathbf{3 . 3 2 2}$ & $\mathbf{2 . 8 1 9}$ & $\mathbf{2 . 2 3 4}$ \\
16 & $\mathbf{3 . 3 8 6}$ & $\mathbf{2 . 8 3 0}$ & $\mathbf{2 . 2 4 1}$ \\
\hline
\end{tabular}


times than for the other two procedures. If we consider UPGMA results only, character 7 ("copepods") had the most substantial effect on the dissimilarities. Its removal shortened the UPGMA tree by 0.131 . The largest increase in tree length $(0.064)$ was caused by the omission of trait 16 ("unidentified animal prey").

Whereas tree length changes appear relatively small, removal of single traits caused comparatively more serious changes in the topology of dendrograms. Changes were drastic in all $16 \mathrm{CL}$ dendrograms, because high level clusters were always modified by removals, and were less substantial for the SL dendrograms. The UPGMA dendrograms were the most robust to trait omissions. These had even less topological changes than SL trees, and for three traits $(4,12$ and 14) the classification topology did not change at all.

\section{Discussion}

Analyses of real data sets demonstrated the utility of Gower's formula in calculating dissimilarities based on species functional traits. This formula applies to a mixture of different measurement scales and tolerates missing values in the data as well. Thus, it has a more general validity than Euclidean distance and related functions also in cases when FD is not dendrogrambased (e.g. quadratic entropy, Botta-Dukát 2005, Ricotta 2005). In Gower's measure, standardization for each variable is implied into the range of [0,1], which means that a priori all functional characters are weighted equally. The range of dissimilarities is also [0,1], thus the coefficient satisfies the requirement postulated by Mason et al. (2005) that FD should not be influenced by the number of traits. In order to select a clustering procedure advocated for further use in calculating dendrogrambased FD, we compared three algorithms: CL, UPGMA and SL. The use of cophenetic correlation hopefully demonstrated for the unconvinced that UPGMA gives a better approximation to the dissimilarities than the other two, consequently UPGMA tree length is also expected to give a more fruitful picture on functional diversity (Blackburn et al. 2005, for similar conclusions).

The results showed that dendrogram length and species number are positively correlated, but this dependence is modified by the inherent multidimensional data structure. Therefore, comparisons between different studies are conclusive only if a higher FD value is obtained for a smaller number of species, a situation detected here for two pairs of studies. In general, FD values coming from different numbers of species could be compared only if some normalization is used to account for the underlying species number effect. We feel that we are far from finding an analytical solution to this problem, and we suggest the use of randomization tests (Manly 1991) if the necessity for such comparisons arises in the future.

Lack of normalization is not a problem, however, when examining the relative rather than the absolute effect of single species removals, because the statistically expected change in FD is the same for all species. Analyses of the intertidal fish data produced the somewhat surprising result that tree length may change in both directions. This shows that total branch length of trees if used as a measure of FD does not satisfy the 'set monotonicity' property (Ricotta 2005) which assumes that diversity should not decrease by the addition of new species. In this sense, the present version of FD bears some relationship to the functional regularity index proposed by Mouillot et al. (2005) which is completely independent of the number of species. Selection of species with most substantial effects on dendrogram length may give us insight regarding their relative influence upon functional diversity. The interpretation we gave in the Examples is valid for the intertidal fish data only, of course, and the explanation of the violation of set monotonicity, if occurs, is always case dependent. In general, the effect of species removal on tree length is a result of the complex interplay between the dissimilarity function and clustering strategy in the modified multidimensional space. If the removed species was in a less extreme position, then the remaining species may fall farther apart in the new data space, thus providing a longer tree. A species with extreme values for a single functional trait appears to contribute much more to the dissimilarities than the others, and its removal leads to a more balanced dissimilarity structure and therefore a shorter tree. Topological changes are always inevitable in such cases, so we did not examine this aspect of the results.

Trait removals may cause both decreases and increases of tree length, but these changes were less substantial in this study than those occurring after species omissions. This result is understandable, because this manipulation does not modify the number of objects, unlike species removals. If the change is negligible, it implies that the given trait adds very little to the dissimilarity-based summary of multidimensional trait structure. The interpretation of relatively large decreases or increases is difficult, however, because the changes result from the combined effect of different modifications of the dendrogram. For instance, tree length may increase because within-cluster distances increase, leaving between-cluster distances unchanged. Increases of FD may appear because the removal of a trait increases between cluster distances, leaving within-cluster distances intact. Whereas clustering procedures differed relatively little for species removal effects on FD, here they were less consistent. Our results support the view that the effect of traits cannot be judged only on the basis of tree length, and the topology of dendrograms 
should also be considered. SL, and especially CL are not robust to trait removals, even though tree length changes are small. Small tree length changes are more proportional to topological changes of UPGMA dendrograms, which is another observation supporting that this clustering method should be used as a standard method for calculating FD. The final conclusion is that the tree length-based definition of FD is a more complex problem than thought before and the examination of the relationship between topological and tree length changes requires future studies.

Acknowledgements - We are grateful to the Subject Editor for useful comments. Financial support from Hungarian Research Grant OTKA T43732 for J. P. is greatly acknowledged. D. S. is a recipient of the Bolyai János Scholarship.

\section{References}

Anderberg, M. R. 1973. Cluster analysis for applications. - Wiley.

Blackburn, T. M., Petchey, O. L., Cassey, P. et al. 2005. Functional diversity of mammalian predators and extinction in island birds. - Ecology 86: 2916-2923.

Botta-Dukát, Z. 2005. Rao's quadratic entropy as a measure of functional diversity based on multiple traits. - J. Veg. Sci. 16: $533-540$

Chapin, F. S. I., Bret-Harte, M. S., Hobbie, S. E. et al. 1996. Plant functional types as predictors of transient responses of arctic vegetation to global change. - J. Veg. Sci. 7: 347358.

Faith, D. P. 1992. Conservation evaluation and phylogenetic diversity. - Biol. Conserv. 61: 1-10.

Golluscio, R. A. and Sala, O. E. 1993. Plant functional types and ecological strategies in Patagonian forbs. - J. Veg. Sci. 4: 839-846.

Gordon, A. D. 1999. Classification, 2nd ed. - Chapman and Hall.

Gower, J. C. 1971. A general coefficient of similarity and some of its properties. - Biometrics 27: 857-874.

Holmes, R. T., Bonney, R. E. J. and Pacala, S. W. 1979. Guild structure of the Hubbard Brook bird community: a multivariate approach. - Ecology 60: 512-520.
Jaksić, F. M. and Medel, R. G. 1990. Objective recognition of guilds: testing for statistically significant species clusters. - Oecologia 82: 87-92.

Manly, B. F. J. 1991. Randomization and Monte Carlo methods in biology. - Chapman and Hall.

Mason, N. W. H., MacGillivray, K., Steel, J. B. et al. 2003. An index of functional diversity. - J. Veg. Sci. 14: 571-578.

Mason, N. W. H., Mouillot, D., Lee, W. G. et al. 2005. Functional richness, functional evenness and functional divergence: the primary components of functional diversity. - Oikos 111: 112-118.

Mouillot, D., Mason, N. W. H., Dumay, O. et al. 2005. Functional regularity: a neglected aspect of functional diversity. - Oecologia 142: 353-369.

Muñoz, A. A. and Ojeda, F. P. 1997. Feeding guild structure of a rocky intertidal fish assemblage in central Chile. - Environ. Biol. Fish. 49: 471-479.

Naeem, S. and Wright, J. P. 2003. Disentangling biodiversity effect on ecosystem functioning: deriving solutions to a seemingly insurmountable problem. - Ecol. Lett. 6: 567579.

Petchey, O. L. and Gaston, K. J. 2002. Functional diversity (FD), species richness and community composition. - Ecol. Lett. 5: 402-411.

Petchey, O. L., Hector, A. and Gaston, K. J. 2004. How do different measures of functional diversity perform? - Ecology 85: 847-857.

Podani, J. 1989. New combinatorial SAHN clustering methods. - Vegetatio 81: 61-77.

Podani, J. 1999. Extending Gower's general coefficient of similarity to ordinal characters. - Taxon 48: 331-340.

Podani, J. 2000. Introduction to the exploration of multivariate biological data. - Backhuys.

Podani, J. 2001. SYN-TAX 2000. Computer programs for data analysis in ecology and systematics. User's manual. - Scientia, Budapest.

Podani, J. 2005. Multivariate exploratory analysis of ordinal data in ecology: pitfalls, problems and solutions. - J. Veg. Sci. 16: 497-510.

Ricotta, C. 2005. A note on functional diversity measures. - Basic Appl. Ecol. 6: 479-486.

Sneath, P. H. A. and Sokal, R. R. 1973. Numerical taxonomy, 2nd ed. - Freeman.

Tilman, D. 2001. Functional diversity. - In: Levin, S. A. (ed.), Encyclopedia of biodiversity. Academic Press, pp. 109-120.

Walker, B., Kinzig, A. and Langridge, J. 1999. Plant attribute diversity, resilience, and ecosystem function: the nature and significance of dominant and minor species. - Ecosystems 2: $95-113$. 